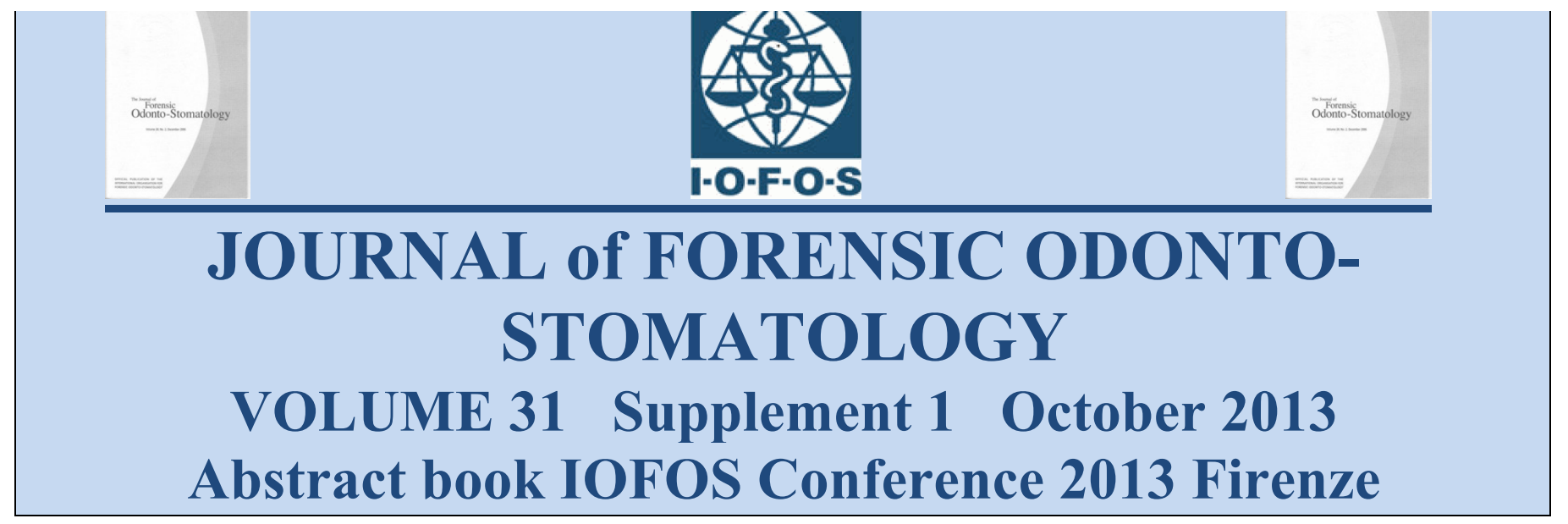

\title{
DENTAL AGE ESTIMATION IN MALAY CHILDREN BASED ON ALL PERMANENT TEETH TYPES
}

Mohd Yusmiaidil Putera Mohd Yusof*, Patrick Thevissen, Steffen Fieuws, Guy Willems

* Centre of Study Oral \& Maxillofacial Diagnostics and Medicine, Faculty of Dentistry, Universiti Teknologi MARA Malaysia

BDS from University of Malaya, Malaysia in 2008. He then worked as a general dentist with the Ministry of Health Malaysia in the northern part of Borneo Island for three years before embarking to new journey as an academia at Department of Oral \& Maxillofacial Diagnostics and Medicine, Faculty of Dentistry, Universiti Teknologi MARA. During his stint with government clinics in Borneo he received and evaluated numbers of disputed age refugees and immigrant workers. In 2012 he completed his master's degree (MSc) in Forensic Odontology at Katholieke Universiteit Leuven, Belgium and he is a professional member of The Forensic Science Society UK (MFSSoc).

The authors declare that they have no conflict of interest.

Background: Since the early study by Demirjian and co-workers in 1973, the Demirjian method has been widely used as a method of choice for dental age estimation. However, due to overwhelming discrepancies in arriving at accurate estimated age by several subsequent studies, Willems and co-workers has modified the statistical method of original study by performing a weighted ANOVA on Belgian children in order to adapt to the scoring system. The purpose of this study was to i) evaluate the applicability of the Willems dental age estimation method in Malay children ii) develop and verify a Malay-specific age prediction model based on the Willems method and iii) to assess the age prediction accuracy by adding third molar information in the Willems model.

Method: Panoramic radiographs ( $n=1403)$ of Malay children aged between 4 and 14.9 years $(n=702)$ and sub-adults aged between 15 and 23.9 years $(n=701)$ were collected. This sample was split in a reference sample to develop a Malay-specific prediction model based on the Willems method and in a test sample to validate this new developed model. The left mandibular seven permanent teeth of the children were scored based on the staging technique described by Demirjian and converted to age using the Willems method. Next, the incorporation of third molars into this model was analyzed. Third molar development of all individuals was staged based on the technique described by Gleiser and Hunt modified by Kohler. Differences between dental age and chronological age were calculated and expressed in mean error (ME), mean absolute error (MAE), and root mean square error (RMSE). 
Results: The Willems model applied on the collected Malay children overestimated chronological age on average $(M E) 0.45$ years $(S D=1.4$ years). Small differences in ME, MAE, and RMSE between the verified Malay-specific prediction model and the Willems model were observed. An overall neglected decrease in RMSE was detected adding third molar stages to the developed permanent teeth model.

Conclusion: Although the Willems model verified on Malaysian children overestimates chronological age, no strong indication was found that warrants the development of a Malaysian-specific prediction model based on a large Malaysian reference sample. Adding agerelated third molar development information to age-related permanent teeth information is only ameliorating the age prediction accuracy in the age group of children between 14 and 16 years.

KEYWORDS: Forensic Odontology, Age estimation, Malay Children, Willem's Method.

JFOS. October 2013, Vol. 31, Sup. No.1, Pag 85-86

ISSN :2219-6749 